\title{
Subjective Determination of Limits of Insurability on the Grounds of Strategic Planning
}

\author{
by Baruch Berliner* and Niklaus Bühlmann*
}

\section{Introduction}

Each professional risk carrier has targets he is aiming at. If he does not wish to rely upon pure chance to drive him towards his targets he must set up a strategic planning to achieve his goals.

In this paper we shall restrict ourselves to strategies involving solely the selection of risks being offered to the professional risk carrier. Such strategies we shall call risk strategies.

One prominent underwriting target that should be included in the risk strategy of any professional risk carrier is the unequivocal distinction between risks that are subjectively insurable for him and risks that are not, taking into account all circumstances.

Since the subjective determination of limits of insurability is a requirement for all reasonable risk strategies we call a risk strategy that fulfils only that requirement restrictive risk strategy. On the other hand we can say that by fixing unequivocally his limits of insurability the professional risk carrier has fixed his restrictive risk strategy. It is better to determine the subjective limits of insurability on the grounds of a restrictive risk strategy than vice versa. The former way leads much more easily, in a more natural way to a systematic, consequent risk policy.

In a further step we shall follow the more ambitious target to select "best" risks out of a set of offers for coverage of insurable risks. By insurable risk we mean a risk that would have been accepted by the professional risk carrier according to his restrictive risk strategy, had it been offered to him alone.

A risk strategy that enables to compare and select risks to achieve ambitious targets like optimizing underwriting results is more extensive than the restrictive risk strategy and should include it. We shall therefore call such a risk strategy an extensive risk strategy.

An extensive risk strategy requires the possibility to compare any two insurable risks. We shall say that the risk strategy gives preference to one risk if it has a higher degree of insurability than another risk.

\footnotetext{
* Swiss Reinsurance Company, Zurich.
} 
We shall introduce nine criteria or components of insurability in such a way that any risk can be decomposed into its nine components. A quantification of the nine components of insurability enables us to quantify risks. As we shall see a quantification of risks does not ensure yet that any two insurable risks are comparable. In a natural way all risks in the subjective domain of insurability of a professional risk carrier are only partially ordered. An extensive risk strategy must therefore be based on one of the nine criteria in a different way than on the other criteria and quantify the risks in such a way that the domain of insurability becomes totally ordered. This will be done later in this paper. The paper will thus show us that a professional risk carrier is bound to certain restrictions when setting up a reasonable extensive risk strategy.

Such an extensive risk strategy will be demonstrated in an example.

\section{Criteria of insurability}

The limits of insurability of a professional risk carrier, be it an insurance or reinsurance company, mutual fund covering risks or a pool, must be fixed according to a certain policy.

Each risk can be decomposed into the same nine components. These components are properties describing the risk. They determine the subjective insurability of a risk for each professional risk carrier.

We therefore call components of a risk - which are independent of any specific risk - criteria of insurability.

The criteria of insurability chosen in [1] are:

(C1) Randomness (of the loss occurrence)

(C2) Maximum possible loss

(C3) Average loss amount upon occurrence

(C4) Average period of time between two loss occurrences

(C5) Insurance premium

(C6) Moral hazard

(C7) Public policy

(C8) Legal restrictions

(C9) Cover limits

We shall call this system of criteria of insurability "the system".

Another criterion which is a function of one or several criteria can replace one of the criteria in the system.

Example: The criterion "loss frequency" can replace the criterion "average period of time between two loss occurrences" in the system. Therefore the two criteria are for us equivalent. If the insurability of a risk is being quantified by assigning numerical values to its components, the two numerical values assumed by the two criteria correspond to the same degree of insurability. By rescaling a quantified criterion to which numerical values have been assigned, we create another criterion which is equivalent to the former one with respect to the insurability of risks.

We also call a quantified criterion of insurability a dimension of insurability. 


\section{Remarks:}

1. The criteria of insurability $(\mathrm{Cl})-(\mathrm{C} 9)$ form a concise and nearly complete system, i.e. in nearly all practical cases they determine unequivocally for each professional risk carrier whether a risk is subjectively insurable for him or not [1].

2. As we restrict ourselves in this paper to risks which are offered for coverage we mean by criterion (C5), "insurance premium", the insurance premium offered to the professional risk carrier.

3. Exceptional cases for which "the system" is not sufficient to determine definitely whether risks are subjectively insurable or not can be constructed.

Example: Let us imagine two risks independent of each other for which all the nine quantified components assume exactly the same values. The only difference between the two risks should be the fluctuations inherent in them. Since they are offered for the same insurance premium the risk subject to greater fluctuations is underrated relative to the other risk. There may therefore exist a professional risk carrier for whom the risk subject to greater fluctuations would be subjectively uninsurable whereas the other risk would be subjectively insurable although both risks are identically quantified in "the system". An unequivocal decision on the subjective insurability of these two risks for all professional risk carriers could only be reached by separating the two risks due to another criterion $(\mathrm{Cl0})$ which we may call "objective fluctuations of risks".

This example is described and discussed more in detail in [1;4.4.1, Remark 3]. The conclusions there are that the example is rather theoretical and that we should refrain for reasons of simplicity from introducing a tenth dimension of insurability in order to better complete "the system".

4. The system (of criteria $(\mathrm{Cl})-(\mathrm{C} 9)$ ) cannot be reduced. Criteria can be replaced by other criteria to form another complete system, but none of the criteria $(\mathrm{Cl})-(\mathrm{C} 9)$ is equivalent to a function of the other eight criteria [1].

5. Some criteria can be decomposed into subcriteria.

We shall adhere, however, to the compound criteria $(\mathrm{Cl})-(\mathrm{C} 9)$ to avoid a too detailed analysis of risk when this is not necessary.

6. Criteria $(\mathrm{Cl})-(\mathrm{C} 9)$ are not independent of each other.

Example: The average loss amount (C3) can never exceed the maximum possible loss (C2).

7. Neither the term "insurability of risks" nor the term "size of a risk" can generally be defined. They can only be analyzed [2]. Consequently, the term "maximum possible loss" has several different meanings in insurance literature. For us it is the sum insured, if this is finite.

\section{A quantification of the criteria of insurability}

In section II we have mentioned the possibility of quantifying the criteria of insurability of "the system", $(\mathrm{Cl})-(\mathrm{C} 9)$, by assigning to each criterion numerical values in a specific interval $I_{k}(k=1, \ldots, 9)$. Each risk is then characterized by nine values assumed by the nine criteria $(\mathrm{Cl})-(\mathrm{C} 9)$. Each of these nine values is called in a geometrical representation a projection of the risk on the respective criterion or axis of insurability. 
In section II we called the projections of a risk components and a quantified criterion of insurability dimension of insurability.

We now plot the dimensions of insurability on 9 orthogonal axes of a nine-dimensional Euclidean space. In this space each risk is quantified by assigning to it one point, respectively a set of nine projections [1].

We aim at a geometrical representation of the criteria of insurability in such a way that all risks corresponding to values i.e. to points inside a boundary area or area of separation are subjectively insurable and all risks corresponding to points outside this area are subjectively uninsurable. When plotting the dimensions of insurability we have to take care that the domain of insurability remains surrounded by the area of separation for each professional risk carrier.

We shall therefore plot the criteria of insurability in such a way that the following definition is valid for each professional risk carrier:

\section{Definition:}

A projection of a risk on an axis is better satisfied than another projection of another risk on the same axis if it assumes a smaller value than the other projection.

We shall moreover require all projections of all risks to assume non-negative values, i.e. each interval $I_{k}$ plotted on axis $k(k=1, \ldots, 9)$ will only assume non-negative values.

Remark: The above definition does not exclude the possibility that for a certain criterion a certain projection of risk $U$ may be better satisfied than that of risk $V$ for one professional risk carrier and vice versa for another. This can happen especially in the case of criteria that are mainly quantified by estimation rather than measurement, such as the criteria (C6), "moral hazard" and (C7), "public policy".

This fact does not disturb us, since we are interested in this paper only in the subjective evaluation of insurability of risks by any professional risk carrier and not in a comparison of such evaluations.

Quantifications of the criteria of insurability:

(Cl): "Randomness" of a risk's loss occurrence is quantified by $(1+\rho) / 2$ where $\rho$ is the correlation coefficient describing the dependence of a risk of all other risks in a portofolio.*

(C2): "Maximum possible loss" is quantified by currency units.

(C3): "Average loss amount upon occurrence" is quantified by currency units.

(C4): "Average period of time between two loss occurrences" is quantified by time units.

(C5): "Insurance premium" we quantify in units of one over the expected loss cost.

(C6): "Moral hazard" is quantified by the degree of moral hazard estimated by the professional risk carrier. No moral hazard is assigned the values zero, absolute moral hazard the value 1 .

(C7): "Public policy" is quantified by the degree of consistency with public policy, estimated by the professional risk carrier. Undoubtful consistency with public policy is assigned the value 0 , undoubtful inconsistency with public policy is assigned the value 1 .

\footnotetext{
* Here we accept negative correlations in a generalization of [1].
} 
Criterion (C8), "legal restrictions", assumes the value 0 for all risks that are legally allowed to be covered and the value 1 for all other risks.

The values assigned to criterion (C9), “cover limits", by the professional risk carrier are values between 0 and 1 , including these two extreme values. They are the closer to value 0 , the more restrictive the cover limits are.

We have now quantified the criteria of insurability in such a way that the domain of insurability is concentrated around the origin and is surrounded by the area of separation which is in turn surrounded by the domain of uninsurability. The intervals $I_{k}$ containing all values of criteria $\left(C_{k}\right)(k=1, \ldots, 9)$ are:

$$
\begin{array}{ll}
I_{1}=[0,1] & I_{6}=[0,1] \\
I_{2}=(0, \infty) & I_{7}=[0,1] \\
I_{3}=(0, \infty) & I_{8}=[0,1] \\
I_{4}=(0, \infty) & I_{9}=[0,1] \\
I_{5}=(0, \infty) &
\end{array}
$$

\section{Strategic planning and risk strategy}

A professional risk carrier must set up rules which his underwriters can follow when deciding whether to grant coverage of a risk.

Definition: A criterion is satisfied for a certain professional risk carrier if it confirms the insurability of a risk offered to him.

The rules should instruct the underwriter if criteria - the values of which may be estimated for an offered risk by himself - are satisfied or not. The rules should be valid for as many risks as possible to avoid time-consuming and often dangerous exceptional cases. In an ideal case the rules should be applied on the same set of criteria in all lines of business. This can be achieved by using a set of criteria which allows to produce an unequivocal decision policy on the subjective insurability of each and every risk. The system of nine criteria of insurability, $(\mathrm{Cl}), \ldots,(\mathrm{C} 9)$ specified and quantified in the last two sections is such a set of criteria for any professional risk carrier.

The underwriting rules of a professional risk carrier should be based on targets.

Such targets could be achieved by means of a short term, medium term and/or long term strategy.

An insurance company's targets for an underwriting year could be, for example:

a) maximizing its expected net profit, given that the standard deviation of the net underwriting result should not exceed $10 \%$ of the net premium income,

b) minimizing its probability of operating loss,

c) minimizing its expected underwriting loss, given that the underwriting result will be negative,

d) minimizing the deviation of the underwriting result from the average of the underwriting results of the last ten years, given a minimum increase of premium income of $10 \%$, 
e) maximizing the premium increase of the company

The objectives of such targets could be

- to demonstrate the company's dynamism,

- to make sure that no decrease in dividends will be necessary,

- to demonstrate the company's stability,

- to secure the company's continued existence etc.

In decision theory we speak of a decision tree or a decision flow diagram including all possible paths from a root, which is the starting point, to a terminal end. On his journey over a certain path the decision maker - which is in our case the professional underwriter - passes chance forks and decision forks that are also called nodes or branching points. The moves from a decision fork are governed by the decision maker, those from a chance fork by chance. A road map on a tree indicates the probability assessements of the various possible branches of each chance fork. They are conditional on the knowledge that would be available to the decision maker at that fork. Moreover, a road map exhibits various costs and the utilities of the various payoffs for following any path to its end.

Given a road map and his partial knowledge and control the decision maker decides at the root on the basis of this strategy which branch to choose at each decision fork he passes on his way to the terminal end [3], [4].

We call the targets the professional risk carrier is aiming at and his strategy to achieve them his strategic planning. The probabilities at chance forks, the assessements of these probabilities and the payoffs at the termini will change in the course of time and may change the terminal end aimed at and the strategy to achieve it. I.e. the strategic planning may change in the course of time.

The strategic planning of a professional risk carrier may be very comprehensive or restrictive depending on his objectives and the targets he sets up to achieve them.

A target of maximizing the operating profit goes for example beyond the responsibility of the underwriters and includes the area of activity of investment specialists.

Once targets have been determined the professional risk carrier must define what it means to "come as close to the targets as possible" in probability terms, in money terms and/ or in other terms. This in turn determines his strategy and the branches he would choose at decision forks following a certain procedure such as averaging out and folding back [4].

We shall restrict ourselves in this paper to the following two forms of strategic planning:

1. Strategic planning involving solely the acceptance respectively turning down of offered risks with the target not to cover subjectively uninsurable risks.

2. Strategic planning involving the selection of risks with the target to select the "best" subset out of a set of risks offered for coverage.

The first form of a strategic planning we shall call a restrictive risk strategy and the second form of strategic planning an extensive risk strategy.

It goes without saying that any extensive risk strategy should include the first form of strategic planning since only subjectively insurable risks should be selected for coverage. 


\section{The restrictive risk strategy}

Restrictive risk strategies include only decision forks with two branches. One branch for the acceptance and the other for the rejection of a risk offered.

A risk strategy restricted to the target not to cover subjectively uninsurable risks is a set of rules determining the boundary area or separation area that separates the domain of insurability, including all subjectively insurable risks, from the domain of uninsurability. Fixing the boundary area means on the other hand the setting up of a restrictive risk strategy. The boundary area includes no existing risks. All risks inside that area are insurable, all risks outside it uninsurable. Underwriters of a professional risk carrier need rules according to which they can act and the professional risk carrier needs rules and targets to control his performance and to optimize his portfolio structure. For a consequent risk policy the area of separation should therefore be fixed on the grounds of a restrictive risk strategy and not vice versa.

If a professional risk carrier decides on insurability by examining whether the 9 criteria of insurability $(\mathrm{Cl})-(\mathrm{C} 9)$ are satisfied or not the first criterion of randomness will depend, for example, on his portfolio's composition. Consequently almost every accepted risk changes slightly the domain of insurability and the area of separation for a professional risk carrier who follows a reasonable restrictive risk strategy.

In the last section we said that the terminal end aimed at in the decision tree may change in the course of time. The tree can be illustrated in a restrictive risk strategy as follows:

Figure 1

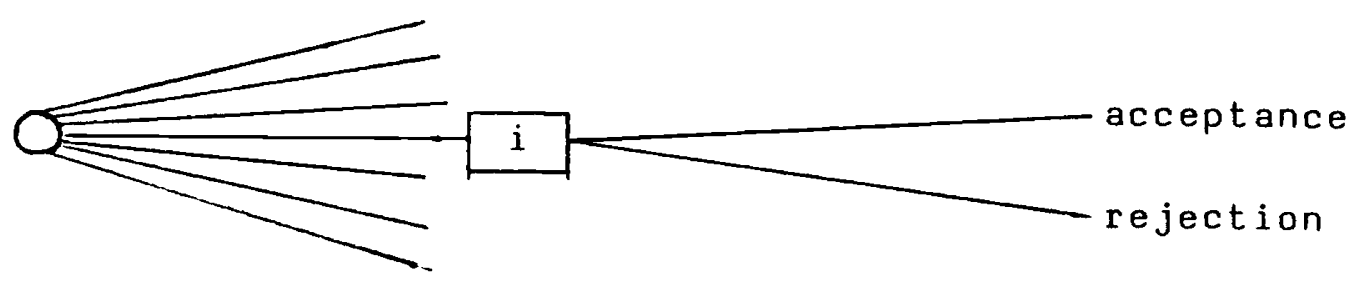

Chance fork of risks offered

Decision fork of risk $i$

The decision on acceptance or rejection of any offered risk may be reversed due to a newly accepted risk. The changing area of separation may have moved one risk or the other from the subjective domain of insurability to the subjective domain of uninsurability or vice versa.

In long term strategy a professional risk carrier's strategic planning should include the objective to enlarge his domain of insurability. Most attempts to improve the position of a professional risk carrier in one way or the other implicity include this objective.

The change of the assessements of probabilities at the chance fork may change the risk carrier's willingness to accept risks, as illustrated in the following example. The necessity to assess probabilities with regard to a risk offered and with regard to potential risks that may be offered makes an experienced underwriter very valuable for a professional risk carrier. 


\section{Example:}

For a given professional risk carrier and portfolio, the same risk may be uninsurable if offered at the beginning of an underwriting year and insurable if offered at year end. At the beginning of the year an underwriter may decline the risk because of other prospective offers which may be more profitable. At year end the probability of such prospective offers may have reduced considerably and an acceptance of the risk that would have been declined months ago may seem opportune.

\section{The subjective domain of insurability}

A point is assigned in the subjective domain of insurability of a professional risk carrier to each existing or hypothetical risk that he would have accepted according to his restrictive risk strategy if it was offered to him. The subjective domain of insurability of the professional risk carrier consist of all such points.

The term "better satisfied" applied in section III on projections we now apply on risks:

\section{Definition}

A risk is better satisfied than another risk if at least one of its projections is better satisfied and none of the latter's projections is better satisfied, i.e. if at least one of its projections assumes a smaller value and none of its projections assumes a larger value than the latter's projections.

We denote two risks by $U=\left(U_{1}, \ldots, U_{9}\right)$ and $V=\left(V_{1}, \ldots, V_{9}\right)$ respectively, where $U_{i}$, $V_{i}$ are the $i$-th projections of risks $U$ and $V(i=1, \ldots, 9)$. If we denote the term "better satisfied" by the relation " $>$ " and the term "at least as well satisfied" by the relation " $\geqslant$ " then

$U \geqslant V \Leftrightarrow U_{i} \leqslant V_{i}$ for all $i=1, \ldots, 9$ and

$U \stackrel{b}{>} V \Leftrightarrow U_{i} \leqslant V_{i}$ for all $i=1, \ldots, 9$ and $U_{k} \neq V_{k}$ for at least one dimension of insurability $\left(C_{k}\right)$, i.e. two different points are assigned to the two risks represented by $U \& V$.

With every point $U$ in the subjective domain of insurability all points $s U(o<s<1)$ which are assigned to risks are better satisfied than $U$ and all points $t \cdot U(t>1)$ which are assigned to insurable risks are worse satisfied than $U$. The larger $t$ is, the closer the point $t \cdot U$ comes to the boundary area. We can say that the domain of insurability is starlike with respect to 0 and with respect to all points corresponding to risks. However, not all points inside the area of separation correspond to risks because for some dimensions of insurability all possible values are discrete and because of negative correlations existing between some criteria of insurability.

\section{Example:}

According to Principle 22 in [1] covering risks for which no real need for insurance exists is inconsistent with public policy and should in principle be avoided. For every individual there exists a limit $M_{\min }$ for a maximum possible loss (Criterion (C2)) below which no necessity for insurance exists. Therefore, the smaller the projection of a risk on axis $(\mathrm{C} 2)$ becomes, the more coverage of the risk should be avoided for reasons of inconsistency with public policy 
(criterion (C7)). Criteria (C2) and (C7) become negatively correlated and their values cannot be jointly reduced for existing risks by reducing, for example, the percentage of participation in a risk.

Denote $\bar{M}_{\min }$ the minimum of $M_{\min }$ for all individuals. For each $M \leqslant \bar{M}_{\min }$ the corresponding risk should be inconsistent with public policy and be therefore uninsurable for every reasonable professional risk carrier.

Therefore, points with a projection $M<\bar{M}_{\min }$ on the second and a small projection on the seventh axis of insurability do not correspond to any existing or hypothetical risk.

We can compare two points in the area of insurability only if all projections of one point are at least as well satisfied as all projections of the other point. This is unsatisfactory. All points with at least one projection being better satisfied and another projection being less well satisfied than the respective projections of point $U$ are not comparable to point $U$.

The space of the dimensions of insurability together with the relation " $\geqslant$ " is partially ordered.

\section{The introduction of a strategic premium}

A restrictive risk strategy is solely concerned with the target not to cover uninsurable risks. All other risks offered would be accepted.

We introduced already in section IV a second form of strategic planning which pursued the much more ambitious target to select the "best" risks out of a set of risks offered for coverage. We called that form of risk planning extensive risk strategy.

For reasons of simplicity we call a percentage of risk $U$ also a risk. $s U$ is a risk with $o<s \leqslant 1$. We thus avoid an introduction of the complex term "partial risk" [1]. Suppose that $U$ is a subjectively insurable risk for a certain professional risk carrier who is offered to cover any reasonable percentage of the risk he prefers to cover. He is thus offered to cover exactly one risk out of the set of risks $\{s U\}, o<s_{o} \leqslant s \leqslant 1$. An extensive risk strategy may select the best risk for coverage out of the set of risks $\{s U\} o<s_{o} \leqslant s \leqslant 1$ whereas a restrictive risk strategy only would confirm that each risk $s U, o<s_{o} \leqslant s \leqslant 1$, is insurable.

This simple example emphasizes the superiority of the extensive risk strategy over the restrictive risk strategy.

However a selection of risks requires the possibility to evaluate any risk and compare it with any other risk with regard to insurability. In the last section we have seen that the naturally evolved term "better satisfied", i.e. the relation $\stackrel{b}{<}$, was partially ordered. It allowed only for comparison of a risk with some other risks.

We now introduce a calculated or strategic premium $\pi(U)$ for risk $U$ as opposed to the offered insurance premium of criterion (C5) that we shall denote $P(U)$. A comparison between $\pi(U)$ and $P(U)$ will allow us to compare any two insurable risks.

With the introduction of a strategic premium we can fulfil a requirement of an extensive risk strategy, namely the target to select the "best" risks out of a set of risks offered for coverage. 
Since we need a strategic premium for every offered risk $U$ an extensive risk strategy must include a premium calculation principle.

In a general case this principle depends on criteria other than (C5) and on loading elements which the professional risk carrier wishes to take into account when calculating the premium for risks.

Such loading elements can be loadings for

- uncertainty due to hazards inherent in the risk,

- uncertainty due to incomplete information on the risk,

- administrative costs,

- services,

- profit, etc.

In remark 3, section II we have revealed the incompleteness of "the system", (Cl), .,, (C9) and mentioned that it could be supplemented by another criterion $(\mathrm{C} 10)$ called "objective fluctuation of risks". In such a completed system of criteria the loading element for "uncertainty due to hazards inherent in the risk" would already be included. We adhere now to "the system" of criteria (Cl), ..., (C9).

Notations:

Risk $U=\left(U_{1}, \ldots, U_{4}\right)$

$$
\begin{aligned}
& U^{-}=\left(U_{1}, \ldots, U_{4}, U_{6}, \ldots, U_{4}\right) \\
& L=\left(l_{1}, \ldots, l_{n}\right), \text { where } \\
& l_{1}=\text { loading element } i, i=1, \ldots n .
\end{aligned}
$$

Definition: A premium calculation principle is a function that assigns to each risk a real, positive number in the following way:

$$
\pi(U):=F\left(U^{-}, L\right) \in R^{+}
$$

$\pi(U)$ is called the strategic or calculated premium for risk $U$.

All components of a risk but the fifth one which is associated with the offered insurance premium are either estimated or determined by the professional risk carrier. Only the insurance premium has the double quality of having an offered value and due to a prenium calculating principle a calculated value.

This double quality allows us to compare any two risks by comparing the two values associated with the insurance premium. We have therefore distinguished the insurance premium from all other criteria of insurability to enable us to define an extensive risk strategy.

A strategic premium should fulfil the following properties:
(a) $\frac{\partial F\left(U^{-}, L\right)}{\partial U_{i}} \geqslant 0 \quad i=1,2,3,6,7,8,9$
(b) $\frac{\partial F\left(U^{-}, L\right)}{\partial U_{4}} \leqslant 0$
(c) $\frac{\partial F\left(U^{-}, L\right)}{\partial L} \geqslant 0$ 
Property (a) symbolizes - also for dimensions that only assume non-continuous values that the strategic premium $\pi(U)$ does not increase with decreasing moral hazard, with decreasing contradiction to public policy or with increasingly severe cover limits.

We call the above properties (a), (b), (c) that are required from a premium calculation principle "reasonable". They bind a professional risk carrier to certain restrictions when fixing an extensive risk strategy, as will be done in section IX.

\section{The degree of subjective insurability}

We call the degree of subjective insurability of risk $U$ the difference between offered and calculated premium.

$$
q(U)=P(U)-\pi(U)=P\left(U_{5}\right)-F\left(U_{1}, \ldots U_{4}, U_{6}, \ldots U_{9}, L\right)
$$

The following properties can be derived for $q(U)$ :

A. Since the offered insurance premium $P\left(U_{5}\right)$ was quantified in section III in units of one over the expected loss cost it follows

$$
\frac{d P\left(U_{5}\right)}{d U_{5}}<0
$$

Therefore we have $\frac{\partial q(U)}{\partial U_{5}}=\frac{d P\left(U_{5}\right)}{d U_{5}}<0$

From properties (a), (b) and (c) of $F\left(U^{-}, L\right)$ follows

B. $\frac{\partial q(U)}{\partial U_{i}} \leqslant 0 \quad$ for $i \neq 4$

C. $\frac{\partial q(U)}{\partial U_{4}} \geqslant 0$

D. $\frac{\partial q(U)}{\partial L} \leqslant 0$

From properties $\mathrm{A}, \mathrm{B}$ and $\mathrm{C}$ follows

E. $\left(U_{i} \leqslant V_{i}, i \neq 4 \& U_{4} \geqslant V_{4}\right) \Rightarrow q(U) \geqslant q(V)$

F. All risks that satisfy the equation

$q\left(U_{1}, \ldots, U_{9}, L\right)=C$, where $C$ is a constant

form a class of risks with the degree $C$ of subjective insurability.

The space of the criteria of insurability is now divided into classes of equal degree of insurability. The set of all classes of equal degree of insurability is totally ordered.

We denote the relation "larger degree of insurability" by " $>$ ". 
Two risks $U, V$ belong to a class of equal degree of subjective insurability if $q(U)=$ $q(V)$. We denote this fact by $U=V . U=V$ neither means here that $U$ and $V$ are the same risk nor that they are represented by the same point.

\section{Conclusions}

We have defined a degree of subjective insurability $q(U)$ for any risk $U$ by considering criterion (C5), insurance premium, to be of special importance among the criteria of insurability. According to the definition of $q(U)$ and property $F q(U)$ fulfills properties we require of a quantitative measurement of risk, namely:

1. $q(U)$ assigns a real number to any risk $U$.

2. Each real constant $C=q(U)$ defines a class of points of invariance of subjective insurability $\{U\}=q^{-1}(C)$ in the space of risks. All risks $U \in\{U\}$ in a class of invariance of subjective insurability have the same degree of subjective insurability and the set of all classes is totally ordered.

$q(U)$ thus makes it possible to compare any two risks. This was not possible before when we defined the initially obvious relation " $\geqslant$ " describing what it means for one risk to be at least as well satisfied as another.

\section{The extensive risk strategy}

Setting up an extensive risk strategy requires the choice of

(a) a reasonable premium calculation principle,

(b) a function

$h\left[q(U), U_{1}, \ldots, U_{4}, U_{6}, \ldots, U_{9}\right]=\hat{h}(U)$ as a measure for the degree of subjective insurability and

(c) a characteristic constant $\hat{C}$ such that

(1) all risks for which $\hat{h}(u)<\hat{C}$ are subjectively uninsurable for the professional risk carrier,

(2) $C^{\prime} \leqslant \hat{C}$ for any constant $C^{\prime}$ for which property (1) holds.

The extensive risk strategy-target of selecting the "best" subset out of a set of risks offered for coverage is achieved by choosing the risks $U$ with the largest measure for degree of subjective insurability $\hat{h}(U)$.

The introduction of a premium calculation principle also enables us to formulate a reasonable restrictive risk strategy more explicitly than in section $\mathrm{V}$ for any professional risk carrier.

By fixing a real constant $\tilde{C}$ and assigning to any existing or hypothetical risk $U$ a strategic premium $\pi(U)=F\left(U^{-}, L\right) \in R^{+}$the risk carrier can achieve the target of a restrictive risk strategy not to cover subjectively uninsurable risks by turning down all offered risks for which

$$
P(U)<\pi(U)+\tilde{C}
$$


$\tilde{C}$ can be positive, negative or equal to zero.

It is convenient to set $\pi(U)=F\left(U^{-}, L\right)=+\infty$ for all risks that would by no means be insured, independent of the premium offered so that $P(U)<\pi(U)+\tilde{C}$.

Example: Criterion (C8), "legal restrictions" assumes the values 1 for all risks that are not legally allowed to be covered. Therefore: $F\left(U_{1}, U_{2}, U_{3}, U_{4}, U_{6}, U_{7}, U_{8}=1, U_{9}, L\right)=\infty$.

\section{Lemma:}

A restrictive risk strategy is a special case of an extensive risk strategy which bases on the same premium calculation principle.

Proof:

We choose

$$
\hat{h}_{0}(U)=\left\{\begin{array}{l}
1 \text { for } q(U)=P(U)-\pi(U) \geqslant \tilde{C} \\
o \text { for } q(U)=P(U)-\pi(U)<\tilde{C}
\end{array}\right.
$$

and $\hat{C}=1$. we have

For all risks for which $P(U)<\pi(U)+\tilde{C}$

$$
\hat{h}_{o}(U)=o<\hat{C}
$$

All these risks are therefore subjectively uninsurable in terms of the chosen extensive risk strategy. q.e.d.

Given an extensive risk strategy with a function $\hat{h}(U)$ and a characteristic constant $\hat{C}$ we can put $\tilde{C}=\hat{C}$ and define a measure for the degree of subjective insurability only for insurable risks by

$$
\hat{h}_{I}(U)=\hat{h}(U) \cdot \hat{h}_{0}(U)
$$

The concept of a restrictive risk strategy is not only included therefore in the concept of an extensive risk strategy as was shown in the last Lemma and required in section IV but it can also be used to construct a concept of an extensive risk strategy that ignores all the subjectively uninsurable risks.

Risk $U$ is preferable to risk $V$ if $\hat{h}(U)>\hat{h}(V)$.

Risks $U$ and $V$ are equivalent with respect to insurability if $\hat{h}(U)=\hat{h}(V)$. Like in property $F$ in the last section we can define totally ordered classes of risks with the same measure for the degree of subjective insurability.

$q(U)$ is an important special case of $\hat{h}(U)$.

A set of offered risks can be ordered

$\hat{h}\left(U^{1}\right)>\hat{h}\left(U^{2}\right)>\ldots$

The larger $\hat{h}\left(U^{i}\right)$ the more preferable is $U^{i}$ for coverage. 
We can divide the task of selecting the "best" set of insurable risks into steps of which each one selects only the "best" risk out of the remaining set of offered risks.

Suppose a professional risk carrier is offered a set of insurable risks $\{U\}$. He first decides to cover the "best" risk $U^{1}$ and is then confronted with the problem of how to choose the "best" risk $U^{2}$ in the set $\left\{U_{-1}\right\}=\{U\}-\left\{U^{1}\right\}$. He goes on to select the "best" risk $U^{3}$ in the set $\left\{U_{-2}\right\}=\left\{\{U\}-U_{i=1}^{2}\left\{U^{i}\right\}\right\}$ and so on. $\left\{U^{i}\right\}$ is the subset of risks that does not remain in the set of risks offered, once the risk $U^{i}$ was chosen for coverage.

Example: In section VII we defined a subset of percentages of risk $U,\{s U\}$, $o<s_{o} \leqslant s \leqslant 1$, out of which the professional risk carrier can only choose one risk $s U$. Once $s U$ is chosen the whole subset $\{s U\}$ does not remain in the set of risks, still offered to the professional risk carrier for coverage.

Each of the risks accepted changes the professional risk carrier's domain of insurability and he stops the procedure of selection with the coverage of risk $U^{k}$ if risk $U^{k+1}$ turns out to be uninsurable.

Following the procedure described above the professional risk carrier has to take only one single decision at each step. This successive selection procedure reduces the extensive risk strategy to manageable dimensions.

\section{An example for an extensive risk strategy}

A professional risk carrier is offered a risk $U$ at a premium $P$. He can also choose to cover $s U$ for $s P$ where $o<s \leqslant 1$. He regards $U$ as subjectively insurable but the question remains: which risk out of the set of risks $\{s U\}$ should he select when following his extensive risk strategy?

Let $T$ be the expected total loss for the period of cover,

$E(x)$ the expected loss amount upon occurrence,

$n$ the expected number of claims during the period of cover,

$M \quad$ the maximum possible loss of risk $U$ upon occurrence and

$\pi \quad$ the premium calculated by the professional risk carrier for coverage of risk $U$.

We assume the distribution function of the total loss to be compound Poisson.

We can introduce a constant $z$, such that

(1) $T=n \cdot E(x)=z \cdot M \quad$ [5] and

(2) $\boldsymbol{\sigma}^{2}=n \cdot E\left(x^{2}\right) \leqslant n E(M \cdot x)=M \cdot n E(x)=z \cdot M^{2}$

If the professional risk carrier applies an approximative variance premium calculation principle, replacing $\boldsymbol{\sigma}^{2}$ by $z \cdot M^{2}$, he arrives at

(3) $\pi=T+c \cdot z M^{2}=T(1+c M)$,

where $c$ is a constant and $\pi$ is the calculated premium for risk $U$ as opposed to the offered premium $P$. 
We call $c \cdot z M^{2}=c T M$ the loading and $c M$ the relative loading. The premium calculation principle (3) is part of the risk carrier's extensive risk strategy.

In formula (3) the premium calculated, $\pi$, is exceptionally a function of criteria (C2), (C3) and (C4) and not of either of the other criteria of insurability nor of any loading element.

The professional risk carrier's strategy is to maximize his profit, i.e. he will select the branch of a decision fork that maximizes the "profit" $q=P-\pi$.

The premium calculation principle described by equation (3) is reasonable, as required in section VII. The measure for the degree of subjective insurability is set $h\left(q(U), U_{1}, U_{6}\right.$, $\left.\mathrm{U}_{7}, U_{8}, U_{9}\right)=h\left(q(u) / U_{1} \leqslant \hat{U}_{1}, U_{6} \leqslant \hat{U}_{6}, U_{7} \leqslant \hat{U}_{7}, U_{8}=0, U_{9} \leqslant \hat{U}_{9}\right)=\hat{h}(U)=q(U)=$ $\left(P-\pi\right.$, provided $U_{1}, U_{6}, U_{7}, U_{8}, U_{9}$ are satisfied) and zero otherwise.

Of the risk $U$ offered the professional risk carrier will thus choose a percentage $s$ such that

$\frac{d}{d s}[(-s T(1+c s M)+s P)]=-T-2 s c M T+P=0$

(4) $1 \geqslant s=\frac{P-T}{2 c \bar{M} T}>0$.

$s U$ is subjectively the "best" risk for the professional risk carrier out of the set of risks $\{s U\}$, where $s$ is calculated in (4).

From (4) we learn that the premium calculation principle (3) and the strategy of maximizing the profit leads to the reasonable principle of participating in the coverage of a risk only as long as the premium offered, $P$, is larger than the expected total loss $T$. This means that in an extensive risk strategy any risk with offered premium $P \leqslant T$ should not be insurable.

The professional risk carrier would accept $100 \%$ of the risk if

$\frac{P-T}{2 c M T} \geqslant 1$, i.e. if

(5) $P \geqslant T(1+2 c M)$ and

(6) $P-\pi \geqslant c M T=c M n E(x)$.

In our example the professional risk carrier would accept $100 \%$ of any risk irrespective of the product of the values assumed by criteria (C2), (C3), (C4) - provided all other criteria of section II are satisfied - if the expected profit divided by constant $c$ exceeds this product according to formula (6).

In other words: According to formula (5) the professional risk carrier would accept $100 \%$ of any risk for which the loading offered is larger or equal to two times the loading calculated and required by him as the "best" risk in the set of risks $\{s U\}$, provided that criteria $(\mathrm{Cl}),(\mathrm{C} 6)$, (C7), (C8) and (C9) are satisfied.

According to a restrictive risk strategy all risks $U$ for which the inequality

$$
P(U) \geqslant \pi(U)+\tilde{C}
$$

is fulfilled are subjectively insurable. 
For $\tilde{C}=-c z M^{2}$ all risks with offered premium

$P(U) \geqslant T$

are insurable.

For $\tilde{C}=O$ only risks with offered premium

$P(U) \geqslant \pi(U)$

are insurable.

In the first case a risk with premium $T \leqslant P<\pi$ would be insurable, in the second case uninsurable.

In case of an extensive risk strategy the "best" risk out of the set of risks $\{s U\}$, $o<s \leqslant 1$ would be for $P=\pi$ following formulas (3) and (4):

$s \cdot U=\frac{T(1+c M)-T}{2 c M T} \cdot U=\frac{U}{2}=50 \%$ of risk $U$.

\section{REFERENCES}

[1] B. Berliner: "Limits of insurability of risks", Prentice-Hall, 1982.

[2] B. Berliner: "Large risks and limits of insurability", The Geneva Paper on Risk and Insurance, Vol. 10, Nr. 37, October 1985.

[3] Bühlmann, Loeffel, Nievergelt: "Entscheidungs- und Spieltheorie", Springer-Verlag, 1975.

[4] H. Raiffa: "Decision analysis, introductory lectures on choices under uncertainty", Addison-Wesley Publishing Company, 1968.

[5] G. Benktander: "A note on profit margin and insurance market capacity", $M V S V M$, Vol. 70, Number $1,1970$. 